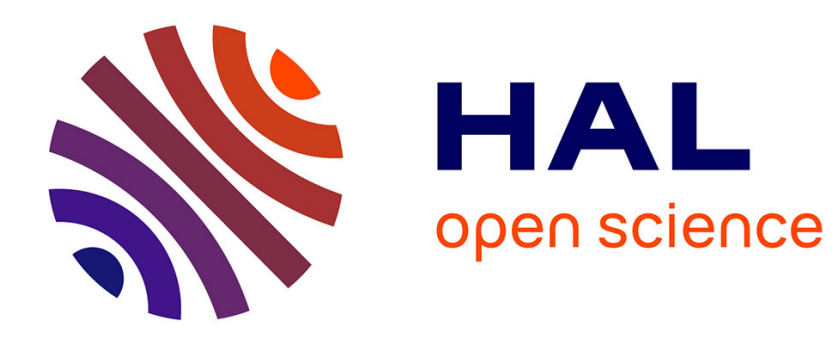

\title{
La canalisation des particules et ses applications
}

\author{
Y. Quéré
}

\section{To cite this version:}

Y. Quéré. La canalisation des particules et ses applications. Revue de Physique Appliquée, 1976, 11

(1), pp.112-112. 10.1051/rphysap:01976001101011200 . jpa-00243950

\section{HAL Id: jpa-00243950 https://hal.science/jpa-00243950}

Submitted on 1 Jan 1976

HAL is a multi-disciplinary open access archive for the deposit and dissemination of scientific research documents, whether they are published or not. The documents may come from teaching and research institutions in France or abroad, or from public or private research centers.
L'archive ouverte pluridisciplinaire HAL, est destinée au dépôt et à la diffusion de documents scientifiques de niveau recherche, publiés ou non, émanant des établissements d'enseignement et de recherche français ou étrangers, des laboratoires publics ou privés. 


\title{
LA CANALISATION DES PARTICULES ET SES APPLICATIONS
}

\author{
Y. QUÉRÉ \\ Section d'Etude des Solides Irradiés \\ Centre d'études nucléaires de Fontenay-aux-Roses, 92260, France
}

\begin{abstract}
Résumé. - La canalisation - ou cheminement privilégié des particules positives entre plans ou rangées atomiques denses d'un cristal - a été longtemps ignorée dans la théorie du cheminement et du ralentissement des particules dans la matière. Découverte au début des années 60 elle a donné lieu depuis à de nombreuses études et applications.

Sa description, du moins pour des particules de masse suffisamment grande (proton, $\alpha \ldots$..., se fait avec une très bonne approximation dans le cadre de la mécanique classique. La particule, tout en progressant le long du canal situé entre plans (ou rangées) atomiques, oscille latéralement. Les principales caractéristiques du mouvement sont liées à cette oscillation et à la diffusion relative à l'axe du canal. Le ralentissement, moindre que celui d'une particule non canalisée, est principalement dû à l'excitation électronique et non aux chocs atomiques.
\end{abstract}

Les applications sont de plusieurs ordres et principalement :

1) Orientation des cristaux. On peut obtenir, à l'aide de faisceaux de particules canalisées, une image du réseau direct (et non du réseau réciproque) et orienter très simplement des cristaux, notamment de taille très réduite $(\simeq 10 \mu)$.

2) Localisation d'atomes étrangers dans la maille. L'étude des particules rétrodiffusées d'un cristal soumis à un bombardement de particules canalisées permet dans certains cas de préciser la position (interstitielle ou substitutionnelle) d'atomes étrangers dans une matrice (impuretés dans $\mathrm{Si}$, métalloïdes ou terres rares dans des métaux cubiques centrés...) ou de certains défauts (complexe impureté-autointerstitiel).

3) Etude de défauts cristallins. Les défauts tendent à décanaliser les particules, soit par pur effet d'obstruction (défaut d'empilement) soit par effet de distorsion (dislocation). L'observation de cette décanalisation peut fournir des indications précieuses notamment sur d'éventuelles inhomogénéités de concentration en défauts (amas de Guinier-Preston par exemple). 